\title{
Disruptive innovators in anaesthesia: data and devices
}

Tong Khee $\underline{\text { Tan, FRCA, LLM }}$

A mong many medical practitioners, anaesthesia as a specialty is a distant experience, perhaps involving attempts as medical students to perform intravenous cannulation and endotracheal intubation. This issue features several articles, selected after rigorous peer review, that highlight the progress made in anaesthesia, in terms of variety of knowledge, specialisation and extent of skills used in patient care. We hope that readers of the Singapore Medical Journal derive some interest and even enlightenment from them.

The concept of big data in healthcare research is not new, although its value is fast gaining recognition. ${ }^{(1)}$ Big data describes the large volume of structured and unstructured data such as patient records, prescription information, treatment plans and disease codes generated within healthcare organisations daily. These encompass the five Vs of big data (volume, variety, velocity, value and veracity). ${ }^{(2)}$ However, it is what health organisations use this data for, rather than its quantity, that matters. Big data can be analysed for insights that lead to better decision-making and enable changes within the healthcare ecology, thereby improving patient care.

The implementation of the Anaesthesia Information Management System (AIMS) has made the practice of manual anaesthesia charting almost obsolete, in keeping with other forms of electronic record-keeping in healthcare services. Using the data captured and through mining information made available through AIMS, Lie et al $^{(3)}$ embarked on a quality improvement (QI) project of investigating compliance and punctuality of administering prophylactic intraoperative antibiotics. AIMS technology was used to create a system to further improve the timeliness of dosing. I have been informed that through the process of data mining, more surgical disciplines were subsequently identified as beneficiaries of this quality initiative.

Lie et al's paper ${ }^{(3)}$ is evidence that rigorous scientific methods can be applied to understand and evaluate the process of QI, thereby helping to ensure that QI efforts are robust, evidencebased and based on best practices, ${ }^{(4)}$ and therefore of sufficient rigour and generalisability to be recognised by peer-reviewed academic journals. Moreover, their paper examined ways to measure, disseminate and replicate their results and successes. ${ }^{(3,5)}$ It showcases how big data is a reality in our health system and the growing importance of improvement science in medicine.(1)

AIMS allows anaesthetists access to accurate and high-quality data. Similar to Lie et al, other researchers have developed systems to capture quality assurance information linked to automated anaesthesia records, resulting in easier, more seamless and betterquality reporting through case documentation. ${ }^{(6)}$ Where do we situate anaesthetists in all this? With their multidisciplinary skill sets, numeracy skills and routine examination of data for trends in clinical settings, they are well suited to lead QI projects within healthcare systems. Their safety culture is particularly useful in many of these contexts.

Conversely, personal experience on the ground shows that much time and attention can be expended when entering data of different degrees of complexity, sometimes in the midst of clinical work. These added work processes can possibly result in diminished attention and potentially distract from patient care. For example, some entries in electronic systems require going into several pages and subpages to find the correct item to click. Moreover, hospitals may use different AIMSs, resulting in the need for relearning with each change of hospital rotation. More importantly, we must consider whether big data and improvement science, on their own, result in behavioural changes and quality enhancement. How much behavioural change is related to incentivisation of staff when they are able to meet their key performance indicators (which are closely aligned with the health system's vision and mission)?

In another variation of technological enhancement in anaesthetic practices, the use of newer airway devices has increasingly helped to avert morbidity and mortality in- and outside of operating theatres. Wong et $\mathrm{al}^{(7)}$ presented a comprehensive review of a topic that is close to the heart of many anaesthetists. Every anaesthetist would have had an unforgettable 'airway patient' or airway-related near miss. This aspect of airway management, in which a patient is not oxygenating well, is hypercarbic and has a trachea that is difficult to intubate, can be extremely stressful for the anaesthetist and even worse for the patient. The advent of supraglottic airway devices has saved many lives over the years; ${ }^{(8)}$ the preponderance of videolaryngoscopes and flexible fibreoptic bronchoscopes has helped to intubate many difficult tracheas. In their article, Wong et $\mathrm{al}^{(7)}$ discussed the indications, contraindications, limitations and complications associated with fibreoptic bronchoscopy. Importantly, they made references to recent airway algorithms (from the United Kingdom's Difficult Airway Society and the American Society of Anesthesiologists ${ }^{(9)}$ and the 4th National Audit Project from the United Kingdom's Royal College of Anaesthetists on major airway complications. ${ }^{(10)}$

Pathways developed by difficult airway societies help to anchor the thought processes of a worried and harried anaesthetist to well-tested algorithms, helping them to make critical airway management decisions rapidly, safely and unequivocally. Wong et $\mathrm{al}^{(7)}$ described various patient groups whose airways can be particularly challenging to the anaesthetist and made suggestions for their management with the aid of fibreoptic 
bronchoscopy. Importantly, the authors reiterated the value of having skills acquisition training on the use of fibreoptic bronchoscopy. Regular training is invaluable because patients with difficult airways do not present frequently and when they do, it should not be the first time an anaesthetist is using certain airway devices and adjuncts.

Personal experience makes me partial to the idea that departments of anaesthesia should always have 'airway champions' who are experts in this aspect of anaesthetic practice. These talents should be highly approachable and supportive of all ranks in matters of airway management, including education, equipment procurement and work processes. When managing airway crises, their presence would be reassuring, their skills are imparted and patients benefit tremendously.

Three other articles in this issue reflect the spirit of inquiry and the role of ultrasonography technology in the quest to improve patient care. Anaesthesia for paediatric surgery is naturally challenging due to the need to manage a range of patients from the very small to the small (premature neonates and older children, respectively) with a wide variety of sometimes unique conditions. In Wong and Lim's article, ${ }^{(11)}$ a formula was derived to guide their practice, relating depth of epidural space to a child's weight in a bid to objectively improve safety. Nonetheless, they declared a caveat of relying on clinical judgement in addition to their formula when assessing the depth to epidural space. We await further studies by these authors to demonstrate the application of their derived formula, particularly its fitness for purpose.

Pain medicine, a specialty born of anaesthesia (at least locally), is so specialised that it warrants a separate department in some hospitals. These feature acute and chronic pain services managed by interprofessional teams. Chutatape et $\mathrm{al}^{(12)}$ answer a question raised by many doctors about the effects of epidural steroids, as well as affirming similar studies. Importantly, the study situates their results in the context of local practice.

Finally, the paper by Lim et al ${ }^{(13)}$ demonstrates an anatomically and functionally specific use for ultrasonography nerve blocks (femoral nerve and adductor canal blocks) as an adjunct analgesic for total knee replacement surgery when minimal motor involvement of quadriceps is an important consideration. This has implications for early mobility of patients after surgery, since femoral nerve blocks can affect the quadriceps, resulting in motor weakness, while adductor canal blocks are predominantly sensory in their effects.

In patients who may be at high risk of complications under general anaesthesia or are too coagulopathic (therapeutically or pathologically) to receive central neuraxial blockade, a properly sited nerve block performed under ultrasonography guidance allows surgery to be performed safely and effectively. This is especially useful for surgery on a peripheral body part such as ray amputation or wound debridement of upper and lower extremities, and can be critical to patient outcome. Previously, there were only several nerve blocks that were performed using landmarks and nerve stimulator localisation of the nerve of interest. With the proliferation and accessibility of portable ultrasonography in point-of-care use, nerve blockades for anaesthesia and analgesia can be more accurately placed. It is interesting that such renewed applications can make anatomy knowledge come alive.

In conclusion, these articles demonstrate a growing culture of scientific enquiry within our health clusters, as investigators and authors admirably seek to answer research questions of interest. The high-quality research questions, methodological alignment and practical applications justify the investigators' efforts. Lim et al's paper ${ }^{(13)}$ was supported by institutional funding, reflecting a support for academic pursuits in tandem with clinical excellence. If publications were no longer a prerequisite for career progression, would we still see such robust expressions of scientific vigour? Perhaps having such a requirement has its virtues, but only if the studies generated fulfil the pragmatism paradigm and produce problem-centred, real world practiceoriented, pluralistic approaches to research questions. ${ }^{(14)}$

\section{REFERENCES}

1. Wagstaff DT, Bedford J, Ramani M. Improvement science in anaesthesia. Curr Anesthesiol Rep 2017; 7:432-9.

2. Ishwarappa, Anuradha J. A brief introduction on big data 5 Vs characteristics and Hadoop technology. Proc Comput Sci 2015; 48:319-24.

3. Lie SA, Lee KY, Goh MH, Harikrishnan S, Poopalalingam R. Achieving 100 percent compliance to perioperative antibiotic administration: a quality improvement initiative. Singapore Med J 2019; 60:130-135.

4. Shojania KG, Grimshaw JM. Evidence-based quality improvement: the state of the science. Health Aff (Millwood) 2005; 24:138-50.

5. The Health Foundation. Evidence scan: Improvement science, January 2011. Available at: https://www.health.org.uk/sites/default/files/ImprovementScience. pdf. Accessed December 26, 2018

6. Peterfreund RA, Driscoll WD, Walsh JL, et al. Evaluation of a mandatory quality assurance data capture in anesthesia: a secure electronic system to capture quality assurance information linked to an automated anesthesia record. Anesth Analg 2011; 112:1218-25

7. Wong J, Lee JSE, Wong TGL, Iqbal R, Wong P. Fibreoptic intubation in airway management: a review article. Singapore Med J 2019; 60:110-118.

8. Ramachandran SK, Kumar AM. Supraglottic airway devices. Respir Care 2014; 59:920-31; discussion 931-2.

9. Frerk C, Mitchell VS, McNarry AF, et al; Difficult Airway Society intubation guidelines working group. Difficult Airway Society 2015 guidelines for management of unanticipated difficult intubation in adults. Br J Anaesth 2015; 115:827-48.

10. Royal College of Anaesthetists, Difficult Airway Society. 4th National Audit Project of the Royal College of Anaesthetists and the Difficult Airway Society. Major complications of airway management in the United Kingdom. Report and findings, March 2011. Available at: http://www.rcoa.ac.uk/system/files/ CSQ-NAP4-Full.pdf. Accessed December 26, 2018.

11. Wong J, Lim SST. Skin-to-epidural distance in the Southeast Asian paediatric population: multiethnic morphometrics and international comparisons. Singapore Med J 2019; 60:136-139.

12. Chutatape A, Menon M, Fook-Chong S, George JM. Metabolic and endocrinal effects of epidural glucocorticoid injections. Singapore Med J 2019; 60:140-144.

13. Lim YC, Quek HYK, Phoo WHJ, Mah CL, Tan S. A randomised controlled trial comparing adductor canal block and femoral nerve block for knee arthroplasty. Singapore Med J 2019; 60:145-149.

14. Creswell JW. Research Design: Qualitative, Quantitative and Mixed Methods Approaches. 3rd ed. Thousand Oaks, CA: Sage Publications Inc, 2009. 